Royal Society; Royal Academy of Engineering, 2004: Nanoscience and nanotechnologies: opportunities and uncertainties. RS policy document 19/04. London, July 2004

Strong, G.; Bainbridge, W.S., 2002: Memetics: A Potential New Science. In: Roco, M.H.; Bainbridge, W.S.: Converging Technologies for Improving $\mathrm{Hu}-$ man Performance: Nanotechnology, Biotechnology, Information Technology and Cognitive Science. http://www.wtec.org/ConvergingTechnologies, pp. 279-286 (17.11.2004)

TAB - Office of Technology Assessment at the German Parliament, 2003: Summary of TAB working report No. 92 "Nanotechnology". http://www.tab.fzk.de/en/projekt/zusammenfassung/ ab92.htm (07.11.2003)

Turkle, S., 2002: Sociable Technologies: Enhancing Human Performance when the Computer is not a Tool but a Companion. In: Roco, M.H.; Bainbridge, W.S.: Converging Technologies for Improving $\mathrm{Hu}-$ man Performance: Nanotechnology, Biotechnology, Information Technology and Cognitive Science. http://www.wtec.org/ConvergingTechnologies, pp. 133-140 (17.11.2004)

Wilsdon, J., 2004: Nanotech needs to listen to its public, and now. Financial Times, 1 September 2004

》

\section{Mobilfunkbranche peilt auf dem Petersberg die Zukunft an}

Zukunftsforum Mobiles Internet 2010, 14. - 15. September 2004

\section{Tagungsbericht von Bernd Wingert und Arnd Weber, ITAS}

Der Ort war gut gewählt, das Gästehaus der Bundesregierung auf dem Petersberg in Königswinter, denn er passte mit seinem prachtvollen Ambiente gut zu der wieder zu Optimismus neigenden Stimmungslage der Mobilfunkbranche. Die Konferenz war Gelegenheit zur Standortbestimmung, zum Rückblick auf bisherige Entwicklungen und das darin Versäumte, wie zum Ausblick auf komplexer werdende Infrastrukturen und ein unübersichtlicher werdendes Feld von Akteuren.

Die Konferenz versammelte weit über 300 Teilnehmer: Mobilfunkbetreiber, Chip- und Gerätehersteller, Dienste- und Inhalteanbieter, auch die universitäre und außeruniversitäre Forschung waren vertreten. Es ging, wie der die Ministerin vertretende Staatssekretär Dudenhausen einleitend betonte, u.a. darum herauszufinden, für welche Ideen und Entwicklungen die im kommenden Jahr zur Verfügung stehenden F+E-Mittel (30 Mio. Euro) eingesetzt werden sollen. Dudenhausen forderte dazu auf, sich die präsentierten Projekte anzusehen, die dann am interessantesten seien, wenn sich Gebiete überkreuzten, wie z. B. Telekommunikation und Nanoelektronik. Die heutige mobile Kommunikation sei erst durch die Nanoelektronik möglich geworden, „und zugleich ist heute die Mobilkommunikation ein Hauptmotor für die Nachfrage nach Nanoelektonik“. Forschung und Industrie sollten enger kooperieren.

Für uns war die Tagung Gelegenheit, eine Zwischenbilanz zu unserem Forschungsprojekt über „i-mode“ (das unter dem Programm für Innovations- und Technikanalysen des BMBF gefördert wird) auf der Postersession zu präsentieren (http://www.itas.fzk.de/deu/projekt/ webe0333c.htm).

\section{Struktur und Themenblöcke}

Die Konferenz ging über zwei Tage; es gab am ersten Tag eine ,Keynote’ von René Obermann (Vorstandsvorsitzender der T-Mobile International AG), und am folgenden Tag sogar zwei Keynotes, die erste von Thomas Ganswindt (Siemens, IuK-Netzwerke), die zweite von Jeffrey Funk (Hitotsubashi University, Tokio). Die Beiträge am Nachmittag des ersten Tages waren den Themen „Infrastruktur“ und „Endgeräte“ gewidmet.

Nach den beiden Keynotes des zweiten Tages waren „Internationale Trends“ Gegenstand der Betrachtung, danach „Anwendungsfelder und Geschäftsmodelle“. Am Nachmittag ging es erneut um Infrastrukturen, nun aber explizit um „Mobile Netze der Zukunft“.

Da es wenig informativ wäre, alle Beiträge mit der gleichen Intensität zu beleuchten, wählen wir aus und sparen die Sektionen über ,Gerätetechnik' und jene zu ,Anwendungen' ganz aus und gehen auch innerhalb der Sektionen nicht auf jeden einzelnen Beitrag ein. 


\section{Infrastruktur: Wie sehen Betreiber und Ausrüster Stand und weitere Entwick- lung?}

Diese Sektion wurde von Gerhard Fettweis geleitet, der an der TU Dresden die Vodafone Stiftungsprofessur innehat. Der einführende Vortrag von René Obermann sei ebenfalls hier subsumiert. Während sich Wiemann (Vodafone) mit der unmittelbar anstehenden Zukunft befasste (der Übergang auf DVB-T und - $\mathrm{H}$, „Digital Video Broadcasting-Terrestrisch bzw. -Handheld") und Möglichkeiten auslotete, welche Marktnischen ein Handy-angepasstes TV besetzen könnte, gingen Wulf (Alcatel, Vorstand Marketing, Vertrieb) und Horn (T-Mobile, Geschäftsführer Technik) auch schon auf die Auslegung künftiger konvergierender Netze ein. Im Vergleich dazu nahmen die Vertreter der beiden Netzbetreiber (Obermann für T-Mobile und Dirks für E-Plus) auch einen kritischen Rückblick vor, der in seiner deutlichen Sprache und Offenheit überraschte. Kritisch kommentiert wurden von Obermann u. a. die unterschätzte Komplexität des mobilen Internet, der Konfigurierungsaufwand für den Nutzer, der nicht immer zufrieden stellend arbeitende Bildtransport, oder der Umstand, dass das Marketing zu lange über die Technologie aufgezogen worden sei und in Zukunft „mehr Kundenverständnis“ aufgebracht werden müsse.

Die Komplexität des mobilen Internet sei, nicht nur von T-Mobile, unterschätzt worden, angefangen bei der Netztechnologie, über die Dienstebereitstellung, die Endgeräte und das Schaffen eines gesamten „Ökosystems“, was wir dahin interpretieren, eine Vielzahl von Beteiligten untereinander abzustimmen (Inhalteanbieter, Netzbetreiber, Handyhersteller). Natürlich sei es gut, wenn die Geräte immer besser würden, aber es stehe, so Obermann wörtlich, ,wenn wir ganz ehrlich sind, in der Nützlichkeit der Dienste, in der Einfachheit der Bedienung und die günstigen Preise, die wir alle brauchen und wollen für den Massenmarkt, noch nicht in allen Bereichen zum Besten.“ Freilich zeigte sich Obermann auch vom Erfolg des mobilen Internet überzeugt.

Wo muss nach Ansicht von René Obermann in Zukunft anders gehandelt werden? Er ging auf fünf Punkte ein: 1) Zwar müsse man alternative Technologien zu UMTS wie WiFi, WiMAX oder OFDM verfolgen, aber sie wür- den in Zukunft ein Netz sehen, das aus verschiedenen Komponenten aufgebaut sei. Das Ganze müsse einfach tarifiert werden. 2) Die Kunden wollten Mobilität und seien bereit, dafür zu bezahlen. Das heutige relativ hohe Preisniveau im Mobilfunk sei aber nicht zu halten. Wichtig sei „Ende-zu-Ende-Qualität“. 3) „Schaffe das mobile Ökosystem!“ war eine weitere Forderung. Man könne in Zukunft nicht mehr alles alleine machen, „walled garden"-Modelle seien nicht mehr aktuell. 4) Man müsse für den Kunden die Komplexität reduzieren, den Techno-Jargon verlassen, die Dienste einfacher machen und auch an die älteren Nutzer denken. 5) Bessere Geräte, mehr Speicher, bessere Displays - das alles sei richtig, aber entscheidend sei, die Gerätesubventionierung herunterzufahren, auch auf die Gefahr hin, dass ein Anbieter dann 1\% Marktanteil verliere. Die Zeit, so Obermann resümierend, wo es um schiere Kundenzahlen ging, sei vorbei. Die Branche müsse sich daran gewöhnen, unter „reifen Marktbedingungen“ zu agieren.

Im kritischen Rückblick auf die bisherige Entwicklung traf sich Thorsten Dirks in vielen Punkten mit René Obermann, so etwa darin, dass die Branche zu technikgetrieben sei und die Kundenorientierung vernachlässigt habe, oder dass die „walled garden“-Strategie in einem mobilen Internet nicht mehr gehen würde. Aber er teilte nicht den Optimismus über den Erfolg von „public hot spots“. Man prüfe zwar auch die neuen Möglichkeiten (wie WiMAX), aber als Technologie im Hintergrund, nicht für die Kundenseite. Was künftige Geschäftsmodelle angeht, plädierte Dirks weder für ein völlig offenes Modell, wo der Betreiber nur als „Pipe“ fungiert, noch für ein völlig geschlossenes. Nach seiner Einschätzung liege das Optimum in der Mitte, also in etwa ,kontrollierte Offenheit'.

\section{Mobiles Internet - was ist das eigentlich?}

In den beiden Keynotes am Mittwochvormittag ging es zum einen schwerpunktmäßig um „machine to machine communication" (von Thomas Ganswindt; Siemens, Bereichsvorstand Information and Communication Networks) und zum anderen um „mobile Internet“ (von Jeffrey Funk, Tokio). Wir konzentrieren uns auf den zweiten Beitrag und schlagen, um das von Obermann Gesagte und das Folgende bes- 
ser einordnen zu können, drei Varianten von „mobilem Internet“ vor.

(1) Eine erste Variante bezieht sich auf die drahtlose Nutzung herkömmlicher InternetDienste, z. B. mittels Laptop oder PDA. Damit kann etwa ein Außendienstmitarbeiter über UMTS oder WiFi Daten aus dem Internet holen kann. Bei dieser Variante kommen Handys noch nicht ins Spiel. (2) Geht es um Angebote wie ,Vodafone live' ,T-Zones' von T-Mobile oder ,i-mode' von E-Plus, dann handelt es sich um Portale der Betreiber, die per Handy zugänglich sind (per Vertrag), um etwa Nachrichten abzurufen oder die aktuelle Wetterkarte anzusehen. Aufgrund des kontrollierten $\mathrm{Zu}-$ gangs nennt man ein solches Konzept „walled garden“. (3) Die dritte Variante liegt vor, wenn Internet-Standards implementiert sind, wie bei „,i-mode“ in Japan, so dass man vom Handy aus direkt eine URL anklicken und die hinterlegte Information abrufen kann (sinnvollerweise für die Bildschirmgröße von Handys angepasst). Diese Variante hat Funk im Auge, wenn er über ,Solving the start up problems in Western mobile Internet markets“ spricht.

Zunächst ging es ihm darum, gängige Vorurteile über den japanischen Mobilfunkmarkt zu zertrümmern, so wenn betont werde, dass die Beschäftigten in Japan sehr viel und lange mit dem Zug fahren, weniger den PC nutzen und Japan eben, auch kulturell, anders sei: „Success of Japan's mobile Internet has nothing to do with trains and low PC Internet usage“.

Für die hohe Handy-Nutzung in Japan sei vor allem entscheidend, was er „push-based Internet Mail“ nannte. Damit werde es möglich, Mails vom PC direkt auf das Handy zu leiten, in die Nachricht eine URL aufzunehmen, die dann wiederum klick- und nutzbar ist, oder es komme zu einer „combination of Internet and other media“.

Das Start-up Problem sieht Funk darin, „to create a critical mass of users and technology providers in industries that have network effects“. Maßnahmen, das ,start up Problem' zu lösen, sind nach Funk u. a. eine Vielzahl von solchen einfachen Anwendungen. Der Markt entstehe nicht dort, wo Nutzer über einen Browser nach Informationen suchten, sondern wo sie sich für spezifische Informationen registrieren lassen, die ihnen per Mail dann zugeht (z. B. dass ein bekannter Künstler dann und dann auf- tritt, so dass gleich ein Ticket bestellt werden kann). Warum würden hiesige Service Provider solche Dienste nicht einrichten?

Funk sieht solche Gründe etwa darin, sich das SMS-Geschäft nicht kaputt zu machen, oder in einem nicht ausreichenden Verständnis der Bedeutung von ,Internet mail’: „In my conversation with service providers I've met very few people who understand the importance of push-based Internet mail. When you talk about mail they think, oh yes, business people want to access their PC mail by their phone. And I say, that's important, but that's not the most important thing. It's for all the other things I've talked about“. Und dazu gehöre auch die Frage, ob Regierungen da nicht Einfluss nehmen sollten. „The mobile Internet is too important to leave it to the service providers ... And so I think that governments need to consider doing some of these things, and the exact way they do them I don't claim to know“.

Es bleibt abzuwarten, ob sich auch die hiesigen Anbieter langfristig auf ein solches offenes Modell zu bewegen werden, wie es Funk skizzierte.

\section{Internationale Trends: DoCoMo in Mün- chen, Siemens in China und WWRF in- ternational}

Hendrik Berndt (Senior Vice President der DoCoMo Euro-Labs in München) befasste sich mit der Architektur von Netzwerken der nächsten Generation (4G). Das von ihnen favorisierte Modell arbeitet mit offenen Plattformen auf jeder Ebene; wichtig seien „programmability \& adaptability“ dieser Netzwerke. Auch Berndt betonte wie andere Referenten (Obermann, Horn, Wulf), dass das Management solcher Netzwerke komplizierter werde. In ihrer Sicht sei 4G eine Kombination von mobiler Welt und einem „ubiquitous networking environment“, das sich gegenüber dem Nutzer auch initiativ verhalten könne. In der Diskussion gefragt, welche Gründe ein japanisches Unternehmen denn bewegen würden, in Deutschland ein Forschungslabor aufzumachen, nannte Berndt u. a. die in Europa und zumal in Deutschland vorhandene lange Tradition von Forschung und die Notwendigkeit, dass DoCoMo im Zuge einer stärker internationalen Positionierung die unterschiedlichen „Räume“ (vermutlich auch als ,Kulturräume’ gemeint) besser verstehen 
müsse. Es gäbe neuerdings auch Labors in den USA und in China.

Im Vortrag von Peter Weiss (Siemens China) wurde von kulturellen Gegebenheiten immer wieder gesprochen, und Siemens müsste Bescheid wissen, sind sie doch schon länger als 100 Jahre in China geschäftlich tätig. Bei Siemens China arbeiten 30.000 Beschäftigte (mit einem Umsatz von 3,3 Mrd. Euro). Ziel sei es, in den kommenden fünf Jahren den Umsatz zu verdoppeln. Weiss erläuterte das Ausbildungssystem (allein im IuK-Bereich 350.000 Absolventen pro Jahr), das forschungspolitische System (im Programm „873“ seien auch Forschungsmittel für „Beyond 3G“ enthalten) und die Telekommunikationsbranche. Sowohl der führende chinesische Festnetz- als auch der Mobilfunkbetreiber sind die größten der Welt. China werde zu einem „lead market“ für den Mobilfunk, im Jahre 2010 rechnet man mit 500 Mio. Mobilfunkteilnehmern, aber der Markt ist noch immer stark durch die Regierung reguliert.

Zwischen China, Korea und Japan entwickeln sich engere Formen der Kooperation, auch um Industriestandards abzusprechen und sich europäischer und amerikanischer Interessen stärker erwehren zu können. Trotz dieses ,Bollwerkes' sieht der Referent eine Chance, sich in diesen Dialog einzubringen. Ihr Ansatz sei, in China für den chinesischen Markt zu produzieren, auch mit guten Ideen und Innovationen, die in Deutschland entstünden. Die Chinesen hätten ,einen unheimlichen Drang, ihr eigener Chef zu sein“, so Weiss in seinem Exkurs über die dortige Mentalität.

Aus nahe liegenden Gründen gab es zu diesem Vortrag viele Nachfragen, so u. a., wie es mit IPR (intellectual property rights) stehe und wie sich Siemens gegen den Diebstahl von Ideen wehre. Hierzu meinte Weiss, eine ,no risk situation“ gebe es nicht; man könne nicht verhindern, dass etwas kopiert werde. Aber das sei nicht entscheidend. Wichtiger sei die Frage, was passiere, wenn sie in diesem Markt überhaupt nicht präsent wären.

Mikko A. Uusitalo erläuterte als Chairman des WWRF (Wireless World Research Forum) Aufgabenverständnis, Ziele und Arbeitsgruppen, Mitglieder und kommende Konferenzen dieser Organisation. Die 150 Mitglieder des WWRF kommen aus allen Kontinenten, wenn auch, wie in der Diskussion klargestellt wurde, „the Euro- pean way of doing" noch dominiere. Die WWRF ist in einem vorwettbewerblichen Bereich tätig und sucht den internationalen Konsens: „Develop a consistent vision of the future Wireless World“, wie eines der Hauptziele umschrieben wurde. Diese Ideen und Modelle sind in einem voluminösen Band zusammengefasst, dem „Book of Visions“. Die Modellvorstellung ist die einer evolutionären Entwicklung hin zu 3G- und 4G-Systemen, nicht eines revolutionären Wandels, der mit „disruptive technologies“ verbunden wäre. Eine solche Gefahr, so Weiss in der Diskussion, sehe er aber in zwei oder drei Jahren aus dem asiatischen Raum heraufziehen. Wir interpretieren diese Hinweise dahin, dass (1) mit neuen Technologien Sprach- und Datendienste billiger als mit UMTS erbracht werden können, (2) neue breitbandige Datennetze, wie sie derzeit in Japan und Korea aufgebaut werden, gemeint sind, wie auch (3) drahtlose Sprachkommunikation über das InternetProtokoll („,voice over IP“).

Dem Thema „Disruptive Technologien in Telekommunikationsnetzen - Verifikation anhand von Prototypen“ war der erste Vortrag von Georg Haubs (Siemens) gewidmet. Der zweite Vortrag von Rainer Fechner (Lucent Technologies Network Systems) befasste sich mit „ABC - Always Best Connected: Konvergenz der Dienste und Systeme“ und betonte den personalisierten Bezug auf einzelne Nutzer und Nutzergruppen. Etwas näher eingehen wollen wir auf Fiona Williams, die über „Towards Ambient Networks" sprach und in Aachen das Ericsson Research Lab aufgebaut hat. Einleitend wies sie darauf hin, dass angesichts der Sättigungskurven in den Industrienationen das kommende Wachstum im Mobilfunk von den Entwicklungsländern erzeugt werde, so dass man gehalten sei, gängige Annahmen zum Bedarf zu überdenken. Konkret malte sie ein Szenario eines mobilen Rockkonzerts aus, das Teil einer Reihe von Projekten im WWIVerbund ist, der ,Wireless World Initiative', die im Kontext des 7. Rahmenprogramms der EU auf eine ,Wireless Communications Technology Platform' hinauslaufen solle, damit Europa mit den ehrgeizigen Aktivitäten von China, Korea und Japan mithalten könne. Forschungsanstrengungen in diesem Bereich seien nötig, denn heute schon gelte, was erst wenige 
so sehen würden: „mobile \& wireless have an economic impact greater than the Internet“.

Bernhard Walke, der Moderator dieser Sektion, lieferte selbst noch einen kleinen Beitrag, bevor er die Diskussion eröffnete. Die Diensteerbringung sei in den heutigen Netzen noch ineffizient. Er schlug ein Konzept vor, nach dem man die mit zunehmender Entfernung von der Basisstation stark abnehmende verfügbare Datenrate für die Nutzer durch Aufstellen von kleinen Relais verbessern kann. Eine solche Komponente sei im WiMAXStandard (IEEE 802.16) als Mesh-Komponente vorgesehen, im Übrigen auf Forschungen basierend, die früher an der TH Aachen gemacht wurden. Deutschland solle die Forschung an Mesh-Konzepten vorantreiben. Das sei eine „disruptive technology“, weil u. a. die Festnetzanschlusskosten dramatisch reduziert werden könnten.

Damit endet unser Einblick in die Konferenz, die auch ein „Zukunftsforum“ sein sollte, und kommen noch einmal auf jenen Ausblick zurück, den man vom Petersberg hat - Modell für den Ausblick in eine ungewisse Zukunft? „Der beste Weg, die Zukunft vorherzusagen ist, sie zu gestalten“ - so zitierte Ganswindt, seinen Vortrag abschließend, Peter Drucker. Gewiss richtig. Nur zeigte die Konferenz gerade an jenen Stellen, wo „disruptive technologies“ angesprochen wurden, wie hoch-kontingent dieses Gestaltungshandeln in einem globalen Kontext geworden ist, wenn mit neuen Techniken die über Jahre gepflegte Technologielinie überrollt zu werden droht.

》

\section{Auf dem Weg zu interdis- ziplinären Methodologien}

Karlsruhe, 24. - 25. Juni 2004

Tagungsbericht von Armin Grunwald, ITAS, und Jan C. Schmidt, Zentrum für Interdisziplinäre Technikforschung, TU Darmstadt

Am 24. und 25. Juni 2004 fand in Karlsruhe der Sondierungsworkshop „Auf dem Weg zu interdisziplinären Methodologien. Forschungsstand und offene Fragen“ statt. Veranstalter waren das Zentrum für Interdisziplinäre Technikforschung an der TU Darmstadt (ZIT) und das Institut für Technikfolgenabschätzung und Systemanalyse des Forschungszentrums Karlsruhe (ITAS).

\section{Fragestellung und Zielsetzung}

Die fortschreitende Differenzierung der Wissenschaften seit der Neuzeit folgte in erster Linie den inneren Bedürfnissen und Entwicklungen der Wissenschaften, weniger den äußeren Anforderungen. In Disziplinen und Subdisziplinen wurden immer spezialisiertere Felder hoch komplizierter Fragestellungen bearbeitet. Das rasche Wachstum und die enorme Leistungssteigerung der Wissenschaft beruht im Wesentlichen auf dieser funktionalen Ausdifferenzierung und Spezialisierung im Wissenschaftssystem.

Im Gegenzug erwartet jedoch die Gesellschaft seit einigen Jahrzehnten verstärkt wissenschaftliche Problemlösungen - z. B. in den Bereichen Umwelt, Energie, Gesundheit -, die sich nicht der disziplinären Spezialisierung fügen. Besondere Bedeutung kommt dabei der Zusammenarbeit zwischen Natur- bzw. Technikwissenschaften einerseits und den „Reflexionswissenschaften“ (Philosophie, Soziologie, Psychologie, Rechtswissenschaften und Ökonomie) andererseits zu. Technikfolgenabschätzung und interdisziplinäre Technikforschung sind einschlägige Gebiete. Inter- und Transdisziplinarität als gesellschaftliche Antwort auf die fortschreitende disziplinäre Spezialisierung der Wissenschaften soll die Wissenschaften wieder stärker an praktischen Problemen der Gesellschaft orientieren und die Parzellierung des Wissens aufheben.

Diese Diskussion um Inter- und Transdisziplinarität dauert seit den siebziger Jahren an. Nach wie vor herrscht der appellative Duktus vor, dass Inter- und Transdisziplinarität dringend benötigt werden. In der Forschungsförderung wird sie häufig erwartet, und in institutionellen Evaluierungen stellt sie mittlerweile meist einen Pluspunkt dar.

In vielen Feldern sind Inter- und Transdisziplinarität mittlerweile fest etabliert, partiell sogar als Teil der Selbstverständnisse von Forschungseinrichtungen oder ganzer Forschungsrichtungen wie z.B. der Nachhaltigkeitsfor- 